\title{
SUPER REGULARITY FOR BELTRAMI SYSTEMS
}

\author{
Gaven J. Martin \\ Massey University, Institute for Advanced Study \\ Auckland, 0632, New Zealand; g.j.martin@massey.ac.nz
}

\begin{abstract}
We prove a surprising higher regularity for solutions to the nonlinear elliptic autonomous Beltrami equation in a planar domain $\Omega$,

$$
f_{\bar{z}}=\mathcal{A}\left(f_{z}\right) \text { a.e. } z \in \Omega,
$$

when $\mathcal{A}$ is linear at $\infty$. Namely $W_{\text {loc }}^{1,1}(\Omega)$ solutions are $W_{\text {loc }}^{2,2+\epsilon}(\Omega)$. Here $\epsilon>0$ depends explicitly on the ellipticity bounds of $\mathcal{A}$. The condition "is linear at $\infty$ " is necessary - the result is false for the equation $f_{\bar{z}}=k\left|f_{z}\right|$, for any $0<k<1,(k=0$ is Weyl's lemma) and the improved regularity is sharp, but can be further improved if, for instance, $\mathcal{A}$ is smooth. We also discuss the subsequent higher regularity implications for fully non-linear Beltrami systems
\end{abstract}

$$
f_{\bar{z}}=\mathcal{A}\left(z, f_{z}\right) \quad \text { a.e. } z \in \Omega,
$$

There the condition "linear at $\infty$ " also implies improved regularity for $W_{\text {loc }}^{1,1}(\Omega)$ solutions.

\section{Introduction and statement of results}

The governing equations of planar geometric analysis and the theory of quasiconformal mappings, Teichmüller spaces and so forth are the Beltrami equations and their nonlinear counterparts, see for instance $[6,15,10,11,13,12]$. Beltrami equations come in several different flavours. As examples, let $\Omega \subset \mathbf{C}$ be a domain and let $f: \Omega \rightarrow \mathbf{C}$ be a mapping of Sobolev class $W_{\text {loc }}^{1,1}(\Omega)$ consisting of functions whose first derivatives are locally integrable. Then we have the following types of Beltrami equations:

- C-linear: $f_{\bar{z}}=\mu(z) f_{z}$, with ellipticity bound $\|\mu\|_{L^{\infty}(\Omega)}<1$;

- R-linear: $f_{\bar{z}}=\mu(z) f_{z}+\nu(z) \overline{f_{z}}$, with ellipticity bound

$$
\||\mu|+|\nu|\|_{L^{\infty}(\Omega)}<1
$$

- Autonomous: $f_{\bar{z}}=\mathcal{A}\left(f_{z}\right)$, with ellipticity bound: there is $k<1$ so that for all $\zeta, \eta \in \mathbf{C}$

$$
|\mathcal{A}(\zeta)-\mathcal{A}(\eta)| \leq k|\zeta-\eta|
$$

- Fully nonlinear: $f_{\bar{z}}=\mathcal{H}\left(z, f, f_{z}\right)$, with ellipticity bound: there is $k<1$ so that for all $z \in \Omega$, all $\zeta, \eta \in \mathbf{C}$

$$
|\mathcal{H}(z, w, \zeta)-\mathcal{H}(z, w, \eta)| \leq k|\zeta-\eta|
$$

with additional conditions on $\mathcal{H}$, see [6, Chapters $7 \& 8]$.

A homeomorphic $W_{\text {loc }}^{1,2}(\Omega)$ solution to any such an equation is a quasiconformal mapping. The theory of planar quasiconformal mappings is comprehensively treated from the perspective of partial differential equations in $[6$, Chapter $7 \& 8]$. An

https://doi.org/10.5186/aasfm.2021.4603

2020 Mathematics Subject Classification: Primary 35J46, 30C62, 35B65.

Key words: Beltrami systems, quasiconformal, higher regularity.

Research supported in part by the New Zealand Marsden Fund. Thanks to two anonymous referees whose careful reading led to significant improvement in exposition. 
orientation-preserving homeomorphism $f: \Omega \rightarrow \Omega^{\prime}$ is $K$-quasiconformal, $1 \leqslant K<\infty$, if $f \in W_{\mathrm{loc}}^{1,2}(\Omega)$ and

$$
\max _{\alpha}\left|\partial_{\alpha} f(z)\right| \leqslant K \min _{\alpha}\left|\partial_{\alpha} f(z)\right|
$$

for almost every $z \in \Omega$.

More generally, any $W_{\text {loc }}^{1,2}(\Omega)$ solution $f$ to any of the above equations is quasiregular and factors as $f=\Phi \circ g$ with $g$ quasiconformal and $\Phi: g(\Omega) \rightarrow \mathbf{C}$ conformal - this is the Stoïlow factorisation theorem, [18].

Each of these equations has a seminal application and they are all inter-related. The apriori assumption that $f \in W_{\mathrm{loc}}^{1,1}(\Omega)$ is so that we can even speak of $f$ as a "solution". Without stronger assumptions on $\mu$ or $\mathcal{H}$ not much can be said, but note for instance that $\mu=0$ on an open set implies $f$ is holomorphic on that set, this is Weyl's Lemma. Another version of Weyl's lemma for C-linear Beltrami equations was given in Theorems 7 and 18 of [9] assuming coefficients in the Sobolev space $W^{1, p}$.

However, the higher regularity theory of these equations typically assumes more on $f$, for instance $f \in W_{\text {loc }}^{1, q}(\Omega)$ for some $1<q \leq 2$ usually depending on the ellipticity constant $k$, and in return delivers a far nicer outcome, $f \in W_{\text {loc }}^{1, p}(\Omega)$ for some $p>2$, again depending on $k$. Astala's theorem [1] gives the optimal result in the $\mathbf{C}$-linear case and can be used to analyse other cases. Questions of existence and uniqueness are fairly well understood through the topological properties of these mappings and Stoïlow factorisation, see also $[6, \S 5.5 \& \S 6.1]$. However there are intriguing subtleties in the nonlinear case, see for instance [3, 4, 5, 14, 17].

Here we present a rather surprising higher regularity theorem for autonomous systems. We say that $\mathcal{A}: \mathbf{C} \rightarrow \mathbf{C}$ is linear at infinity if there are constants $a, b \in \mathbf{C}$, $|a|+|b|<1$, and $\alpha$ with $0 \leq \alpha<1$, such that

$$
\mathcal{A}(\zeta)=a \zeta+b \bar{\zeta}+O\left(|\zeta|^{\alpha}\right)
$$

The next theorem makes no ellipticity assumptions on $\mathcal{A}$.

Theorem 1. (Super-regularity for autonomous systems) Let $\Omega \subset \mathbf{C}$ be a planar domain and $f: \Omega \rightarrow \mathbf{C}$ be a $W_{\text {loc }}^{1,1}(\Omega)$ solution to the autonomous Beltrami system

$$
f_{\bar{z}}=\mathcal{A}\left(f_{z}\right), \quad \text { a.e. } z \in \Omega,
$$

where $\mathcal{A}$ is linear at $\infty$. Then $f \in W_{\text {loc }}^{1, p}(\Omega)$ for all $p<\infty$. If, in addition, $|\mathcal{A}(\zeta)| \leq k|\zeta|$ for some $k<1$ and all $\zeta \in \mathbf{C}$, then $f$ is also quasiregular.

For nonlinear systems, the proof of Theorem 1 provides us with the following. General results concerning the existence, uniqueness and regularity of the solutions to the nonlinear elliptic equations

$$
\mathcal{F}(z, f, \nabla f)=0
$$

for mappings $g: \Omega \rightarrow \mathbf{C}$ in two dimensions. Section 7.7 of [6] shows that under the assumption of ellipticity, such systems reduce to

$$
\frac{\partial f}{\partial \bar{z}}=\mathcal{H}\left(z, f, \frac{\partial f}{\partial z}\right)
$$

The existence theory for such systems can be established under surprisingly general conditions even in the nonlinear setting, see [6, Chapter 8]. When one is looking for solutions to these general nonlinear elliptic systems there are necessarily constraints on $\mathcal{H}$. In [6] these are for $\mathcal{H}: \mathbf{C} \times \mathbf{C} \times \mathbf{C} \rightarrow \mathbf{C}$, 
1. The homogeneity condition, that $f_{\bar{z}}=0$ whenever $f_{z}=0$, equivalently,

$$
H(z, w, 0) \equiv 0, \quad \text { for almost every }(z, w) \in \mathbf{C} \times \mathbf{C}
$$

2. The uniform ellipticity condition, that for almost every $z, w \in \mathbf{C}$ and all $\zeta, \xi \in \mathbf{C}$,

$$
|H(z, w, \zeta)-H(z, w, \xi)| \leqslant k|\zeta-\xi|, \quad 0 \leqslant k<1
$$

3. $H$ is Lusin-measurable: Thus

- There are compact $Z_{1} \subset Z_{2} \subset \cdots \subset \mathbf{C}$ whose union has full measure.

- There are compact $W_{1} \subset W_{2} \subset \cdots \subset \mathbf{C}$ whose union has full measure.

- There are compact $\Xi_{1} \subset \Xi_{2} \subset \cdots \subset \mathbf{C}$ whose union has full measure.

- For each $j=1,2, \ldots$, the map $H: Z_{j} \times W_{j} \times \Xi_{j} \rightarrow \mathbf{C}$ is continuous.

As a consequence of our Theorem 1 we have the following.

Theorem 2. Suppose that $\mathcal{H}: \Omega \times \Omega^{\prime} \times \mathbf{C} \rightarrow \mathbf{C}$ satisfies the conditions above with ellipticity bound $k$ and is also linear at $\infty$ on the $W_{\text {loc }}^{1,1}(\Omega)$ solution $f$. That is there are constants $a, b \in \mathbf{C}$ such that $|a|+|b|<1$ and

$$
\left|\mathcal{H}\left(z, f, f_{z}\right)-a f_{z}-b \overline{f_{z}}\right| \in L_{\mathrm{loc}}^{q}(\Omega), \quad \text { for some } q>1+k .
$$

Then in fact $f \in W_{\text {loc }}^{1, s}(\Omega)$ for all $s<1+1 / k$. Hence $f$ is quasiregular.

In the next corollary we should realise that there is no assumed connection between $|a|+|b|$ and $k$. However in applications it will be obvious that typically $|a|+|b| \leq k$.

Corollary 1. Suppose that $f \in W_{\text {loc }}^{1,1}(\Omega)$ is a solution to (3) for $\mathcal{A}$ linear at $\infty$, and $f$ satisfies a Lipschitz bound of the form

$$
\left|f_{\bar{z}}(z+t \zeta)-f_{\bar{z}}(z)\right| \leq k\left|f_{z}(z+t \zeta)-f_{z}(z)\right|, \quad|\zeta|=1,
$$

for some $k<1$ and for all $0<t<a(z)$ for some continuous function $a: \Omega \rightarrow \mathbf{R}_{+}$, $a(z) \leq \operatorname{dist}(z, \partial \Omega)$. Then:

1. $f \in W_{\text {loc }}^{2, q}(\Omega)$ for all $q<1+1 / k$.

2. Each member of the $\mathbf{R}$-linear family

$$
\left\{a f_{x}(z)+b f_{y}(z): a, b \in \mathbf{R}\right\}
$$

is either $\frac{1+k}{1-k}$-quasiregular mapping or a constant.

3. There are measurable $\mu, \nu: \Omega \rightarrow \mathbf{C}$ with $|\mu|+|\nu| \leq k$ so that both directional derivatives $f_{x}$ and $f_{y}$ satisfy the $\mathbf{R}$-linear Beltrami equation,

$$
h_{\bar{z}}=\mu(z) h_{z}+\nu(z) \overline{h_{z}}, \quad h \in\left\{f_{x}, f_{y}\right\} .
$$

4. The complex gradient $f_{z}$ is itself quasiregular and satisifies the $\mathbf{R}$ linear equation

$$
h_{\bar{z}}=\frac{\mu(z)}{1-|\nu(z)|^{2}} h_{z}+\frac{\overline{\mu(z)} \nu(z)}{1-|\nu(z)|^{2}} \overline{h_{z}}, \quad h=f_{z}
$$

and thus $f_{z} \in W_{\text {loc }}^{1, q}(\Omega)$ for all

$$
q<s=1+1 / k^{\prime}, \quad k^{\prime}=\left\|\frac{|\mu|}{1-|\nu|}\right\|_{\infty} \leq k .
$$


Of course the Lipschitz bound at (8) is achieved if for instance $\mathcal{A}$ satisfies the usual ellipticity assumption: $\mathcal{A}$ is $k$-Lipschitz,

$$
|\mathcal{A}(\zeta)-\mathcal{A}(\eta)| \leq k|\zeta-\eta|, \quad \zeta, \eta \in \mathbf{C} .
$$

In this case one can make further claims about existence, uniqueness and higher regularity of $W_{\text {loc }}^{1,2}(\Omega)$ solutions $[6,3,5,7]$. An example of these which follows from the Schauder theory would be the following.

Corollary 2. Let $\mathcal{A}: \mathbf{C} \rightarrow \mathbf{C}$ be smooth and linear at $\infty$ with

$$
\left|A_{\zeta}(\eta)\right|+\left|A_{\bar{\zeta}}(\eta)\right|<1, \quad \eta \in \mathbf{C} .
$$

Then every $W_{\text {loc }}^{1,1}(\Omega)$ solution $f$ to the equation (3) is smooth and quasiregular. After appropriate normalisation a homeomorphic $f$ will be unique.

Indeed [4] characterised characterised the condirions of Corollary 1 and established surprising improved Schauder regularity properties for solutions to the LerayLions divergence type equation in the plane via nonlinear Beltrami equations.

In contrast to these results however is another very interesting result of Astala et al. [2] which shows the condition of being linear at $\infty$ is essential.

Theorem 3. For each $k<1$ there exists an $f \in W_{\text {loc }}^{1,1}(\mathbf{C})$ solving the equation

$$
f_{\bar{z}}=k\left|f_{z}\right|
$$

for which $f \notin W_{\text {loc }}^{1,1+k}(\mathbf{C})$. Any solution to (11) which lies in $W_{\text {loc }}^{1, q}(\mathbf{C})$ for some $q>1+k$ is smooth.

\section{Proofs}

The main result, Theorem 1, and its corollaries are a consequence of an induction based on the following result.

Theorem 4. Let $\mathcal{A}: \mathbf{C} \rightarrow \mathbf{C}$ be linear at $\infty$ and $h \in L_{\text {loc }}^{q}(\Omega)$. Then every $W_{\text {loc }}^{1,1}(\Omega)$ solution to the equation

$$
f_{\bar{z}}=\mathcal{A}\left(f_{z}\right)+h
$$

lies in $W_{\mathrm{loc}}^{1, q}(\Omega)$.

Proof. Let $\eta \in C_{0}^{\infty}(\Omega)$. We write (12) as

$$
f_{\bar{z}}=a f_{z}+b \overline{f_{z}}+O\left(\left|f_{z}\right|^{\alpha}\right)+h
$$

for some $\alpha<1$. We multiply this equation by $\eta$ and use the fact that

$$
(\eta f)_{\bar{z}}=\eta f_{\bar{z}}+\eta_{\bar{z}} f, \quad(\eta f)_{z}=\eta f_{z}+\eta_{z} f
$$

and rearrange terms to achieve the following equation for $\tilde{f}=\eta f$.

$$
\tilde{f}_{\bar{z}}=a \tilde{f}_{z}+b \overline{\tilde{f}_{z}}+u
$$

where

$$
u=\eta h+\eta O\left(\left|f_{z}\right|^{\alpha}\right)+\eta_{\bar{z}} f+a \eta_{z} f+b \overline{\eta_{z} f}
$$

The Sobolev embedding gives $\eta f \in L^{r}(\mathbf{C})$ for every $r<2$ and so we can assume $u \in L^{1 / \alpha}(\mathbf{C}) \cap L^{q}(\mathbf{C}) \cap L^{r}(\mathbf{C})$ for every $r<2$. We therefore have to show that any compactly supported $W^{1,1}(\mathbf{C})$ solution to the inhomogeneous constant coefficient equation (13) has improved regularity. If $\mathcal{S}$ denotes the Beurling transform, a singular integral operator of Calderon-Zygmund type, since $\mathcal{S} \circ \frac{\partial}{\partial \bar{z}}=\frac{\partial}{\partial z}$ we really want to 
establish the invertibility of the constant coefficient Beltrami operator $I-a \mathcal{S}-b \overline{\mathcal{S}}$ since (13) reads as

$$
\tilde{f}_{\bar{z}}-a \mathcal{S}\left(\tilde{f}_{\bar{z}}\right)-b \overline{\mathcal{S}\left(\tilde{f}_{\bar{z}}\right)}=u
$$

More on Beltrami operators can be found in [6, Chapter 4].

Following $[6, \S 15.2]$ we address (13) by a linear change of variables reducing it to the inhomogeneous Cauchy-Riemann equation. Namely, for any given constants $\mu, \nu \in \mathbf{D}$ we may use the transformation

$$
\tilde{f}(\zeta)=\frac{g(z)-\nu \overline{g(z)}}{1-|\nu|^{2}}, \quad \zeta=z+\mu \bar{z},
$$

which rearranges to

$$
g(z)=\tilde{f}(\zeta)+\nu \bar{f}(\zeta), \quad z=\frac{\zeta-\mu \bar{\zeta}}{1-|\mu|^{2}}
$$

and we set $v(z)=u(\zeta)=u(z+\mu \bar{z})$. If we make the following choices for $\mu$ and $\nu$,

$$
\begin{aligned}
& \mu=\frac{-2 a}{1+|a|^{2}-|b|^{2}+\sqrt{\left(1+|a|-|b|^{2}\right)^{2}-4|a|^{2}}}, \\
& \nu=\frac{-2 b}{1+|b|^{2}-|a|^{2}+\sqrt{\left(1+|b|-|a|^{2}\right)^{2}-4|a|^{2}}},
\end{aligned}
$$

then since $|a|+|b|<1$ some computation reveals that indeed

$$
|\mu| \leqslant \frac{|a|}{1-|b|^{2}}<1, \quad|\nu| \leqslant \frac{|b|}{1-|a|^{2}}<1 .
$$

Thus we can make these transformations above and a few further elementary computations shows that (13) now reads as

$$
\frac{\partial g}{\partial \bar{z}}(z)=v(z)+a b \overline{v(z)}
$$

Hence $g_{\bar{z}} \in L^{1 / \alpha}(\mathbf{C}) \cap L^{q}(\mathbf{C}) \cap L^{r}(\mathbf{C})$ and since $\mathcal{S}: L^{q}(\mathbf{C}) \rightarrow L^{q}(\mathbf{C})$ is bounded, we have $g_{z}=\mathcal{S}\left(g_{\bar{z}}\right) \in L^{1 / \alpha}(\mathbf{C}) \cap L^{q}(\mathbf{C}) \cap L^{r}(\mathbf{C})$, note that the equation $g_{z}=\mathcal{S}\left(g_{\bar{z}}\right)$ additionally uses the decay of $g$ to be valid. Thus $g$ (and hence $\tilde{f}$ and consequently $f$ ) lies in the space $W_{\text {loc }}^{1, s}(\Omega)$, where $s=\min \{1 / \alpha, q, r\}$. Iterating this construction we achieve $s=q$ as $\alpha \rightarrow \alpha^{2}$ and $r$ increases to the new Sobolev embedding exponent.

Now Theorem 1 is immediate using the result of Theorem 4, and Corollary 1 also follows as Theorem 1 promotes $f$ to an element of $W_{\text {loc }}^{1,2}(\Omega)$ (actually better) and so we may appeal to the main result of [12]. As for Corollary 2, the only remark that needs to be made is that if $\mathcal{A}$ is smooth with the proposed bounds, and linear at $\infty$, then $\mathcal{A}$ must satisfy some Lipschitz bound with constant less than 1 . Corollary 2 then put the solution in $W_{\text {loc }}^{2,2}(\Omega)$, the complex gradient is quasiregular, and so Hölder continuous. Then, as noted, the Schauder bounds [6] give the desired conclusion. Actually Lemma 3.1 of the [5] gives some details about how to express $h_{\bar{w}}$ as a function of $h_{w}$ are given in the general non-autonomous case.

\section{A remark on the Hodographic transform}

It is interesting to note that under the Hodographic transformation $[6, \S 16.3]$ (basically a change of variables for homeomorphic solutions) and under a few regularity 
assumptions we see a relationship between conditions on $f$ and $h=f^{-1}$. The condition "linear at $\infty$ " is simply that there is $q>1$ such that $\left|f_{\bar{z}}-a f_{z}-b \overline{f_{z}}\right| \in L_{\text {loc }}^{q}(\Omega)$. If $h$ is sufficiently regular so as to change variables we find

$$
\left|f_{\bar{z}}(h)-a f_{z}(h)-b \overline{f_{z}(h)}\right| J(w, h)=\left|h_{\bar{w}}-b h_{w}-a \overline{h_{w}}\right|
$$

should be an $L_{\mathrm{loc}}^{q}\left(\Omega^{\prime}\right)$ function. That is essentially the same condition, though notice that $h$ will satisfy the equation

$$
h_{\bar{w}}=J(w, h) \mathcal{A}\left(\frac{h_{w}}{J(w, h)}\right) .
$$

It is not obvious how one unravels this equation to express $h_{\bar{w}}$ as a function of $h_{w}$ other than locally using the implicit function theorem, but note that $\left|h_{\bar{w}}\right|=$ $J(w, h)\left|\mathcal{A}\left(\frac{h_{w}}{J(w, h)}\right)\right| \leq k\left|h_{w}\right|$ still holds.

\section{Fully nonlinear Beltrami systems}

We now discuss the immediate consequences for the fully nonlinear Beltrami system and the proof for Theorem 2. Thus we consider the equation

$$
f_{\bar{z}}=\mathcal{H}\left(z, f, f_{z}\right)
$$

with ellipticity conditions that there is $k<1$ so that for all $z \in \Omega, \zeta \in \Omega^{\prime}$ and all $\zeta, \eta \in \mathbf{C}$

$$
|\mathcal{H}(z, w, \zeta)-\mathcal{H}(z, w, \eta)| \leq k|\zeta-\eta|
$$

$\mathcal{H},(z, \eta, 0) \equiv 0$, with additional measurability condition $\mathcal{H}(z, w, \eta): \Omega \times \Omega^{\prime} \times \mathbf{C} \rightarrow \mathbf{C}$ is Lusin measurable, see [6, Chapters $7 \& 8]$ for the most general requirements. Roughly, the condition of Lusin measurability assures us that the function is measurable in each variable independently.

Now Theorem 2 can be established as follows. We may suppose $q<2$. Put $u(z)=\mathcal{H}\left(z, f, f_{z}\right)-a f_{z}-b \overline{f_{z}}$. Our hypotheses put $u \in L_{\mathrm{loc}}^{q}(\Omega)$ and we observe $f_{\bar{z}}=a f_{z}+b \overline{f_{z}}+u(z)$. The proof of Theorem 4 (without using induction) puts $f \in W_{\text {loc }}^{1, q}(\Omega)$. Then Astala's theorem [1] implies that $f \in W_{\text {loc }}^{1, s}(\Omega)$ and the ellipticity bound gives us this for all $s<1+1 / k$. Observe that $1+1 / k>2$.

However, using an induction to improve regularity offers other alternative results for the nonlinear case. We formulate one such now. Suppose that the ellipticity and measurability conditions following (17) hold. Specifically:

(1) There is $k<1$ so that for all $z \in \Omega, \zeta \in \Omega^{\prime}$ and all $\zeta, \eta \in \mathbf{C}$

$$
|\mathcal{H}(z, w, \zeta)-\mathcal{H}(z, w, \eta)| \leq k|\zeta-\eta| .
$$

(2) $\mathcal{H},(z, \eta, 0) \equiv 0$,

(3) $\mathcal{H}(z, w, \eta): \Omega \times \Omega^{\prime} \times \mathbf{C} \rightarrow \mathbf{C}$ is Lusin measurable,

(4) That there are $a, b \in \mathbf{C},|a|+|b| \leq k$ and

$$
\mathcal{H}(z, \eta, \zeta)=a \zeta+b \bar{\zeta}+U(z, \eta, \zeta)
$$

and there is $\alpha<1$ and $u \in L_{\mathrm{loc}}^{q}(\Omega)$ for some $q>1+k$ such that

$$
|U(z, \eta, \zeta)| \leq A|\zeta|^{\alpha}+B|\eta|^{2 \alpha}+u(z) \text {. }
$$

Theorem 5. Let $f: \Omega \rightarrow \Omega^{\prime}$ be a $W_{\text {loc }}^{1,1}(\Omega)$ solution to the nonlinear equation (17) satisfying (1)-(4) above. Then $f \in W_{\text {loc }}^{1,2}(\Omega)$ is a $\frac{1+k}{1-k}$-quasiregular mapping. 
The proof is basically the same. Initially we have $f \in L_{\text {loc }}^{q}(\Omega)$ for all $q<2$ by Sobolev embedding. So the first two terms will self improve in an induction. The last term sets the limit of the improvability of the Sobolev exponent, but with the ellipticity bound and Astala's theorem this will be enough to guarantee quasiregularity. There are other ways to reformulate the bounds on condition(19) so as to achieve a similar result. We leave these for the reader to consider.

\section{References}

[1] Astala, K.: Area distortion of quasiconformal mappings. - Acta Math. 173, 1994, 37-60.

[2] Astala, K. Personal communication. - 2019.

[3] Astala, K., A. Clop, D. Faraco, J. JäÄskeläinen, and A. Koski: Nonlinear Beltrami operators, Schauder estimates and bounds for the Jacobian. - Ann. Inst. H. Poincaré Anal. Non Linéaire 34, 2017, 1543-1559.

[4] Astala, K., A. Clop, D. Faraco, J. JäÄskeläinen, and A. Koski: Improved Hölder regularity for strongly elliptic PDEs. - https://arxiv.org/pdf/1906.10906.pdf.

[5] Astala, K., A. Clop, D. Faraco, J. JäÄskeläinen, and L. SzÉKelyhidi: Uniqueness of normalized homeomorphic solutions to nonlinear Beltrami equations. - Int. Math. Res. Not. IMRN 18, 2012, 4101-4119.

[6] Astala, K., T. Iwaniec, and G. J. Martin: Elliptic partial differential equations and quasiconformal mappings in the plane. - Princeton Mathematical Series 48, Princeton Univ. Press, Princeton, NJ, 2009.

[7] Astala, K., and J. JÄÄSKeläInen: Homeomorphic solutions to reduced Beltrami equations. - Ann. Acad. Sci. Fenn. Math. 34, 2009, 607-613.

[8] Bojarski, B., L. D'Onofrio, T. Iwaniec, and C. Sbordone: G-closed classes of elliptic operators in the complex plane. - Ric. Mat. 54, 2005, 403-432.

[9] Clop, A., D. Faraco, J. Mateu, J. Orobitg, and X. Zhong: Beltrami equations with coefficient in the Sobolev space $W^{1, p}$. - Publ. Mat. 53, 2009, 197-230.

[10] Gardiner, F.P.: Teichmüller theory and quadratic differentials. - Pure Appl. Math. (New York), John Wiley \& Sons, Inc., New York, 1987.

[11] Gardiner, F.P., and N. Lakic: Quasiconformal Teichmüller theory. - Math. Surveys Monogr. 76, Amer. Math. Soc., Providence, RI, 2000.

[12] Hinkkanen, A., and G. Martin: Quasiregular families bounded in $L^{p}$ and elliptic estimates. - J. Geom. Anal. (to appear).

[13] Hubbard, J. H.: Teichmüller theory and applications to geometry, topology, and dynamics. Teichmüller theory, volume 1, Matrix Editions, Ithaca, NY, 2006.

[14] Iwaniec, T., and C. Sbordone: Quasiharmonic felds. - Ann. Inst. H. Poincaré Anal. Non Linéaire 18, 2001, 519-5-72.

[15] Iwaniec, T., and G. J. Martin: The Beltrami equation. - Mem. Amer. Math. Soc. 191, 2008.

[16] Martin, G. J., and C. YaO: $L^{p}$-Teichmüller theory. - arXiv:2007.15149 [math.CV], 2020.

[17] Meyers, N.: An $L^{p}$-estimate for the gradient of solutions of second order elliptic divergence equations. - Ann. Sc. Norm. Super. Pisa 17, 1963, 189-206.

[18] SтоїLоw, S.: Brief summary of my research work. - Analysis and Topology 1-7, World Sci. Publ., River Edge, NJ, 1998.

[19] Struwe, M.: Variational methods. Applications to nonlinear partial differential equations and Hamiltonian systems. Fourth edition. - Ergeb. Math. Grenzgeb. (3) 34, Springer-Verlag, Berlin, 2008. 